\section{Myocardial protection: is there anything better than ice?}

B.A. Finegan MB BCH FRCPC, M. Cohen MD
$\mathrm{T}$

HE classical method of protecting the heart from the consequences of ischaemia during open heart surgery, cold blood cardioplegic arrest, is no longer considered by many to provide optimal results. Warm cardioplegic arrest ${ }^{1}$ and coronary artery surgery without the aid of cardiopulmonary bypass ${ }^{2,3}$ are not uncommon in many centres. The use of tissue plasminogen activator and/or urgent angioplasty has revolutionised the non-surgical treatment of acute coronary occlusion. ${ }^{4}$ These clinical developments have occurred in consort with an enhanced understanding of the mechanisms underlying myocardial reperfusion-induced cell injury. ${ }^{5,6}$ A number of novel pharmacological and non-pharmacological strategies aimed at enhancing myocardial protection prior to ischaemia and at limiting injury on reperfusion following ischaemia are under investigation. This review details the mechanisms and clinical experience to date of four such interventions of particular interest to the author.

\section{Altering energy substrate metabolism}

The heart is an omnivore capable of using free fatty acids, glucose, lactate and pyruvate as energy substrates. ${ }^{7}$ Under normal aerobic conditions, free fatty acids constitute the main energy source for the heart. Complete oxidation of the 16 - carbon fatty acid palmitate for example, produces 129 molecules of the energy storage molecule adenosine triphosphate (ATP), with an ATP yield per oxygen atom taken up of 2.83 . Glucose, the other major fuel used by the heart, is metabolised initially to pyruvate (a process termed glycolysis), which enters the citric acid cycle in the form of acetyl $\mathrm{CoA}$ and undergoes complete oxidation to $\mathrm{CO}_{2}$ and $\mathrm{H}_{2} \mathrm{O}$. The decarboxylation of pyruvate to acetyl $\mathrm{CoA}$ is catalysed by the pyruvate dehydrogenase complex (PDC) Aerobic disposal of glucose yields 36 molecules of ATP, with an ATP yield per oxygen atom taken up of 3.17. In the absence of $\mathrm{O}_{2}$, pyruvate is converted to lactate (anaerobic glycolysis) yielding under these circumstances only two molecules of ATP per molecule of glucose entering the glycolytic pathway.
During no-flow ischaemia the heart relies entirely on anaerobic metabolism of glucose for ATP production. The source of glucose during no-flow ischaemia is the glucose storage molecule, glycogen. A molecule of glycogen metabolised to lactate, produces three molecules of ATP. The marked difference between energy production under aerobic and anaerobic conditions underscores the urgency of restoring blood flow and thereby oxidative metabolism to ischaemic myocytes. Also the $\mathrm{O}_{2}$ sparing effect of glucose relative to free fatty acids suggests that preferential use of glucose during reperfusion following ischaemia should be beneficial. Enhancing glucose oxidation on reperfusion following ischaemia has been shown to improve ventricular function in a number of in vitro studies. ${ }^{8,9}$

Although glycolysis is essential during no-flow ischaemia, it is a two-edged sword that has the potential to be damaging if not appropriately modulated during ischaemia and reperfusion. When glycolytically produced ATP is used within the cell, $\mathrm{H}^{+}$ions are produced. The cell exchanges $\mathrm{H}^{+}$ions for $\mathrm{Na}^{+}$via the $\mathrm{Na}^{+} / \mathrm{H}^{+}$exchanger in an effort to maintain cellular $\mathrm{pH}$. Consequently, during ischaemia, but particularly on reperfusion, there is a net gain of $\mathrm{Na}^{+}$ions within the cell. ${ }^{10}$ Increased intracellular $\mathrm{Na}^{+}$activates the $\mathrm{Na}^{+} / \mathrm{Ca}^{++}$exchange mechanism. ${ }^{11}$ The entry of excess $\mathrm{Ca}^{++}$has been implicated as one of the key processes mediating myocardial injury. Increased intracellular $\mathrm{Ca}^{++}$evokes increased cycling of $\mathrm{Ca}^{++}$in and out of the sarcoplasmic reticulum and increased mitochondrial storage of $\mathrm{Ca}^{++}$both ATP wasting processes, triggers phospholipase activation and consequently cell membrane damage. ${ }^{5}$

Under conditions of stress, as during cardiac surgery or following a myocardial infarction, free fatty acid levels are increased and glucose oxidation is inhibited. ${ }^{12}$ This uncoupling of glucose oxidation from glycolysis is potentially harmful as it increases $\mathrm{H}^{+}$ion production and reduces efficient ATP production. Altering myocardial substrate metabolism during ischaemia by preventing excessive glycolysis and during reperfusion, by enhancing glucose oxidation and improving the coupling

From the Department of Anaesthesia, University of Alberta, Edmonton. Alberta, Canada T6G 2El. E-mail: bfinegan@gpu.srv.ualberta.ca 
between glucose oxidation and glycolysis improves functional recovery following ischaemia in animal models of global ischaemia. ${ }^{13}$ Clinical studies in patients suffering from ischaemic heart disease of ranolazine, a drug which stimulate glucose oxidation by enhancing PDC activity, have shown that while ranolazine improves diastolic function in patients who have suffered a previous myocardial infarction ${ }^{14}$ it does not appear to be effective in reducing the incidence or severity of angina. ${ }^{15}$ The role of PDC stimulants in the reducing ischaemia-reperfusion injury in man is still under investigation.

\section{Ischemic preconditioning}

The phenomenon of ischaemic preconditioning (IPC), a process whereby brief, repetitive episodes of ischaemia induce temporary protection against injury resulting from a subsequent more prolonged period of ischaemia was first described by Murry et al. ${ }^{16}$ in 1986. This important protective mechanism is associated with a reduction in infarct size following regional ischaemia, ${ }^{17}$ enhanced recovery of mechanical function during reperfusion following global no-flow ischaemia ${ }^{18}$ and attenuation of ischaemia-induced dysrhythmias. ${ }^{19}$ IPC improves the coupling between glucose oxidation and glycolysis and protects the heart from the consequences of global no-flow ischaemia. ${ }^{18}$ While ischaemia is the classical preconditioning stimulus it is now apparent that many other interventions can also precondition the heart. Considerable evidence has accumulated to implicate adenosine as a trigger or mediator of IPC..$^{20,21}$ Adenosine $A_{1}$ receptor stimulation mimics $I P C^{22}$ and the protective effect of IPC is reduced by Ado receptor antagonists ${ }^{17}$ and facilitated by either increasing the release of Ado, ${ }^{23,24}$ or by reducing the inactivation of Ado. ${ }^{25}$ Other mechanisms of IPC, including increased heat shock protein synthesis, ${ }^{26}$ enhanced ATP-dependant $\mathrm{K}^{+}$channel activity, ${ }^{27}$ reduced ATP utilisation, ${ }^{16}$ and increased protein kinase $\mathrm{C}$ activity $^{28}$ have also been proposed. IPC-like protection has also been observed following bradykinin, and catecholamine administration. ${ }^{28,29}$

IPC protocols in patients undergoing angioplasty are associated with a reduction in the incidence of ventricular ectopy during coronary occlusion ${ }^{30}$ and reduction in the magnitude of ST-segment alterations during balloon inflation. ${ }^{31}$ Similarly, in patients undergoing open heart surgery an IPC protocol comprising of two three-minute periods of aortic cross-clamping and an intervening two minute period of reperfusion followed by ten minutes of ischaemia demonstrated a slower rate of ATP depletion and troponin $T$ release compared with control hearts. ${ }^{32}$ In cardiac surgical "off pump" coronary revascularisation procedures,
IPC is frequently performed on the target vessel to provide protection during the no-flow period of surgical anastomosis.

\section{Adenosine}

Endogenous and exogenous administered adenosine protects the heart from the consequences of ischaemia with marked reductions in infarct size $\mathrm{e}^{33}$ and improved recovery of mechanical function during reperfusion being observed in animal models of regional ${ }^{34}$ and global ischaemia. ${ }^{13}$

Adenosine may act by multiple protective pathways. These include coronary and collateral vessel dilation (that increase $\mathrm{O}_{2}$ supply), modulation of the activity of catecholamines, protein kinase $\mathrm{C}$ activation, opening $\mathrm{K}+\mathrm{ATP}$ channels (that cause negative inotropic and chronotropic actions and decrease $\mathrm{O}_{2}$ demand), and inhibition of neutrophil cytotoxicity and platelet adherence in the coronary microcirculation. ${ }^{35}$ Adenosine under aerobic conditions inhibits glycolysis, enhances the coupling between glycolysis and glucose oxidation and reduces $\mathrm{H}^{+}$ production arising from glucose metabolism. ${ }^{13}$

There is, to date, one clinical trial published investigating the effects of adenosine administration in patients undergoing heart surgery. Mentzer et al. ${ }^{36}$ noted a reduction in inotropic requirements and an improved ejection fraction in patients who received adenosine $140 \mu \mathrm{mol} \cdot \mathrm{kg}^{-1} \cdot \mathrm{min}^{-1}$ immediately prebypasss and cold blood cardioplegic solutions supplemented with $500 \mu \mathrm{M}$ to $2 \mathrm{mM}$ adenosine compared with placebo controls. Adenosine administration was also associated with significantly reduced blood and blood product requirements. ${ }^{37}$ In patients undergoing angioplasty, a $10-\mathrm{min}$ intracoronary infusion of adenosine $\left(2 \mathrm{mg} \cdot \mathrm{min}^{-1}\right)$ prior to percutaneous transluminal angioplasty significantly reduced ST-segment shift and chest pain associated with initial balloon inflation compared to control placebo-treated subjects. ${ }^{38}$ These exciting preliminary data suggest that adenosine pretreatment may be appropriate in elective cardiac procedures where the heart is subjected to ischaemia-reperfusion events.

\section{Anaesthetic agents}

Volatile anaesthetics, in particular halothane and isoflurane, appear to possess cardioprotective properties. In animal models of regional and global ischaemia, either or both agents, if present prior or during the ischaemic episode, improve post-ischaemic left ventricular function, ${ }^{39}$ limit myocardial stunning, ${ }^{40}$ decrease reperfusion induced dysrhythmias ${ }^{41}$ and reduce infarct size. ${ }^{42}$ The effectiveness of isoflurane and halothane if present only during reperfusion in limiting ischaemic dysfunction is less certain. ${ }^{43}$ 
Halothane decreases myocardial contractility and oxygen consumption to a greater extent than isoflurane or enflurane, without altering coronary flow or oxygen delivery ${ }^{44}$ Isoflurane increases coronary flow and hence oxygen delivery with limited effects on contractility at less than one MAC concentrations. ${ }^{45}$ Volatile agents exert similar effects on oxygen supply-demand balance in humans with coronary artery disease. ${ }^{46}$ Volatile anaesthetics preserve high energy phosphate levels in the myocardium. Halothane improves recovery of myocardial ATP content upon reperfusion, ${ }^{47}$ decreases lactate release during ischaemia and decrease the breakdown of ATP to adenosine and inosine. ${ }^{48}$ Isoflurane also preserves myocardial ATP content in hearts subject to ischaemia and reperfusion. ${ }^{49}$ There is strong evidence that volatile anaesthetic drugs may aid the myocardium in normalising the pathological $\mathrm{Ca}^{++}$cycling during reperfusion. Halothane, in particular, decreases myocyte $\mathrm{Ca}^{++}$accumulation ${ }^{50}$ directly reducing release of $\mathrm{Ca}^{++}$from the sarcoplasmic reticulum ${ }^{51}$ and inhibiting the $\mathrm{Na}^{+} / \mathrm{Ca}^{++}$ exchange mechanism to prevent $\mathrm{Ca}^{++}$influx. ${ }^{52}$

\section{Conclusion}

The enhanced understanding of the cellular responses to ischaemia and reperfusion following ischaemia has allowed the development of many new and exciting strategies to limit the adverse consequences of prolonged myocardial ischaemia. Clinical application of this knowledge offers hope of improved outcomes for patients suffering from coronary artery disease.

\section{References}

1 Mauney $M C$, Kron $I L$. The physiologic basis of warm cardioplegia. Ann Thorac Surg 1995; 60: 819-23.

2 Mack MJ, Acuff TE, Casimir-Abn H, Lonn UJ, Jansen $E W$. Video-assisted coronary bypass grafting on the beating heart. Ann Thorac Surg 1997; 63: S100-3.

3 Rivetti LA, Gandra SM. Initial experience using an intraluminal shunt during revascularization of the beating heart. Ann Thorac Surg 1997; 63: 1742-7.

4 Sweeney JP, Schwartz GG. Applying the results of large clinical trials in the management of acute myocardial infarction. Western Journal of Medicine 1996; 164: 238-48.

5 Karmazyn $M$. The 1990 Merck Frosst Award. Ischemic and reperfusion injury in the heart. Cellular mechanisms and pharmacological interventions. Can J Physiol Pharmacol 1991; 69: 719-30.

6 Hansen PR. Role of neutrophils in myocardial ischemia and reperfusion. Circulation 1995; 91: 1872-85.
7 Ferrari $R$. Metabolic disturbances during myocardial ischemia and reperfusion. Am J Cardiol 1995; 76: 17B-24B.

8 McVeigh J, Lopaschuk GD. Dichloroacetate stimulation of glucose oxidation improves recovery of ischemic rat hearts. Am J Physiol 1990; 259: H1079-85.

9 Lewandowski ED, White LT. Pyruvate dehydrogenase influences postischemic heart function. Circulation 1995; 91: 2071-9.

10 Lazdunski $M$, Frelin $C$, Vigne $P$. The sodium/hydrogen exchange system in cardiac cells: its biochemical and pharmacological properties and its role in regulating internal concentrations of sodium and internal $\mathrm{pH}$. J Mol Cell Cardiol 1985; 17: 1029-42.

11 Anderson SE, Cala PM, Steenbergen C, London RE, Murphy E. Effects of hypoxia and acidification on myocardial $\mathrm{Na}$ and $\mathrm{Ca}$. Role of $\mathrm{Na}-\mathrm{H}$ and $\mathrm{Na}-\mathrm{Ca}$ exchange. Ann N Y Acad Sci 1991; 639: 453-5.

12 Lopaschuk GD, Collins-Nakai R, Olley PM, et al. Plasma fatty acid levels in infants and adults after myocardial ischemia. Am Heart J 1994; 128: 61-7.

13 Finegan BA, Lopaschuk GD, Coulson CS, Clanachan $A S$. Adenosine alters glucose use during ischemia and reperfusion in isolated rat hearts. Circulation 1993; 87: 900-8. 14 Hayasbida W, pan Eyll C, Rousseau $M F$, Pouleur $H$. Effects of ranolazine on left ventricular regional diastolic function in patients with ischemic heart disease. Cardiovasc Drugs Ther 1994; 8: 741-7.

15 Thadani $U$, Ezekowitz $M$, Fenney $L$, Chiang $r K$. Double-blind efficacy and safety study of a novel antiischemic agent, ranolazine, versus placebo in patients with chronic stable angina pectoris. Ranolazine Study Group. Circulation 1994; 90: 726-34.

16 Murry $C E$, Jennings $R B$, Reimer $K A$. Preconditioning with ischemia: a delay of lethal cell injury in ischemic myocardium. Circulation 1986; 74: 1124-36.

17 Liu GS, Thornton J, Van Winkle DM, Stanley AW, Olsson RA, Downey JM. Protection against infarction afforded by preconditioning is mediated by $\mathrm{Al}$ adenosine receptors in rabbit heart. Circulation $1991 ; 84$ : 350-6.

18 Finegan $B A$, Lopaschuk $G D$, Gandhi $M$, Clanachan AS. Ischemic preconditioning inhibits glycolysis and proton production in isolated working rat hearts. Am J Physiol 1995; 269: H1767-75.

19 Hagar JM, Hale SL, Kloner RA. Effect of preconditioning ischemia on reperfusion arrhythmias after coronary artery occlusion and reperfusion in the rat. Circ Res 1991; 68: 61-8.

20 Downey JM, Coben MV, Ttrebus $K$, Liu $\Upsilon$. Cellular mechanisms in ischemic preconditioning: the role of adenosine and protein kinase C. Ann N Y Acad Sci 1994; 723: 82-98. 
21 Miura $T$, Iimura $O$. Infarct size limitation by preconditioning: its phenomenological features and the key role of adenosine. Cardiovasc Res 1993; 27: 36-42.

22 Finegan BA, Lopaschuk GD, Gandhi M, Clanachan AS. Inhibition of glycolysis and enhanced mechanical function of working rat hearts as a resulat of adenosine adenosine $A_{1}$ recertor stimulation during reperfusion following ischaemia. Br J Pharmacol 1996; 118: 355-63.

23 Tsucbida A, Yang XM, Burckbartt B, Mullane KM, Coben $M V$, Downey JM. Acadesine extends the window of protection afforded by ischaemic preconditioning. Cardiovasc Res 1994; 28: 379-83.

24 Tsuchida A, Liu GS, Mullane K, Downey JM. Acadesine lowers temporal threshold for the myocardial infarct size limiting effect of preconditioning. Cardiovasc Res 1993; 27: 116-20.

25 Miura T, Ogawa T, Iwamoto T, Shimamoto K, Iimura $O$. Dipyridamole potentiates the myocardial infarct sizelimiting effect of ischemic preconditioning. Circulation 1992; 86: 979-85.

26 Liu $X$, Engelman RM, Moraru II, et al. Heat shock. A new approach for myocardial preservation in cardiac surgery. Circulation 1992; 86: II358-63.

27 Gross GJ, Auchampach JA. Blockade of ATP-sensitive potassium channels prevents myocardial preconditioning in dogs. Circ Res 1992; 70: 223-33.

$28 \mathrm{Hu} K$, Nattel S. Mechanisms of ischemic preconditioning in rat hearts. Involvement of alpha 1B-adrenoceptors, pertussis toxin-sensitive $\mathrm{G}$ proteins, and protein kinase C. Circulation 1995; 92: 2259-65.

29 Linz W, Wiemer G, Scholkens BA. Beneficial effects of bradykinin on myocardial energy metabolism and infarct size. Am J Cardiol 1997; 80: 118A-23A.

30 Airaksinen KE, Huikuri HV. Antiarrhythmic effect of repeated coronary occlusion during balloon angioplasty. J Am Coll Cardiol 1997; 29: 1035-8.

31 Cribier A, Korsatz $L$, Koning $R$, et al. Improved myocardial ischemic response and enhanced collateral circulation with long repetitive coronary occlusion during angioplasty: a prospective study. J Am Coll Cardiol 1992; 20: 578-86.

32 Alkhulaifi AM. Preconditioning the human heart. Ann R Coll Surg Engl 1997; 79: 49-54.

33 Toombs CF, McGee S, Johnston WE, Vinten Johansen J. Myocardial protective effects of adenosine. Infarct size reduction with pretreatment and continued receptor stimulation during ischemia. Circulation 1992; 86: 986-94.

34 Pitarys CJ, Virmani R, Vildibill HDJ, Jackson EK, Forman $M B$. Reduction of myocardial reperfusion injury by intravenous adenosine administered during the early reperfusion period. Circulation 1991; 83: 237-47.

35 Ely SW, Berne RM. Protective effects of adenosine in myocardial ischemia. Circulation 1992; 85: 893-904.
36 Mentzer RM Jr, Rabko PS, Molina-Viamonte $V$, et al. Safety, tolerance, and efficacy of adenosine as an additive to blood cardioplegia in humans during coronary artery bypass surgery. Am J Cardiol 1997; 79: 38-43.

37 Mentzer RM Jr, Rabko PS, Canver CC, et al.

Adenosine reduces postbypass transfusion requirements in humans after heart surgery. Ann Surg 1996; 224: 523-9; discussion 529-30. 38 Leesar MA, Stoddard $M$, Abmed $M$, Broadbent $J$, Bolli R. Preconditioning of human myocardium with adenosine during coronary angioplasty. Circulation 1997; 95: 2500-7.

39 Coetzee A, Moolman J. Halothane and the reperfusion injury in the intact animal model. Anesth Analg 1993; 76: 734-44.

40 Warltier DC, al-Wathiqui $M H$, Kampine JP, Schmeling $W T$. Recovery of contractile function of stunned myocardium in chronically instrumented dogs is enhanced by halothane or isoflurane. Anesthesiology 1988; 69: 552-65.

41 Buljubasic N, Marijic J, Stowe DF, Kampine JP, Bosnjak ZJ. Halothane reduces dysrhythmias and improves contractile function after global hypoperfusion in isolated hearts. Anesth Analg 1992; 74: 384-94.

42 Davis RF, Sidi $A$. Effect of isoflurane on the extent of myocardial necrosis and on systemic hemodynamics, regional myocardial blood flow, and regional myocardial metabolism in dogs after coronary artery occlusion. Anesth Analg 1989; 69: 575-86.

43 Belo SE, Mazer CD. Effect of halothane and isoflurane on postischemic "stunned" myocardium in the dog. Anesthesiology 1990; 73: 1243-51.

44 Sahlman L, Henriksson BA, Martner J, Ricksten SE. Effects of halothane, enflurane, and isoflurane on coronary vascular tone, myocardial performance, and oxygen consumption during controlled changes in aortic and left atrial pressure. Studies on isolated working rat hearts in vitro. Anesthesiology 1988; 69: 1-10.

45 Stowe DF, Marijic J, Bosnjak ZJ, Kampine JP. Direct comparative effects of halothane, enflurane, and isoflurane on oxygen supply and demand in isolated hearts. Anesthesiology 1991; 74: 1087-95.

46 Khambatta HJ, Sonntag $H$, Larsen $R$, Stephan H, Stone $J G$, Kettler D. Global and regional myocardial blood flow and metabolism during equipotent halothane and isoflurane anesthesia in patients with coronary artery disease [see comments]. Anesth Analg 1988; 67: 936-42.

47 Coetzee A, Brits W, Genade S, Lochner A. Halothane does have protective properties in the isolated ischemic rat heart. Anesth Analg 1991; 73: 711-9.

48 Buljubasic N, Stowe DF, Marijic J, Roerig DL, Kampine $J P$, Bosnjak ZJ. Halothane reduces release of adenosine, inosine, and lactate with ischemia and reperfusion in isolated hearts. Anesth Analg 1993; 76: 54-62. 
49 Kanaya N, Kobayashi I, Nakayama $M$, Fujita S, Namiki $A$. ATP sparing effect of isoflurane during ischaemia and reperfusion of the canine heart. $\mathrm{Br}$ J Anaesth 1995; 74: 563-8.

50 Hoka S, Bosnjak ZJ, Kampine JP. Halothane inhibits calcium accumulation following myocardial ischemia and calcium paradox in guinea pig hearts. Anesthesiology 1987; 67: 197-202.

51 Komai $H, R$ usy BF. Direct effect of halothane and isoflurane on the function of the sarcoplasmic reticulum in intact rabbit atria. Anesthesiology 1990; 72: 694-8.

52 Haworth RA, Goknur AB. Inhibition of sodium/calcium exchange and calcium channels of heart cells by volatile anesthestics. Anesthesiology 1995; 82: 1255-65. 
B.A. Finegan MB BCH FRCPC, M. Cohen MD

\section{Protection myocardique: $y$ a-t-il mieux que la glace?}

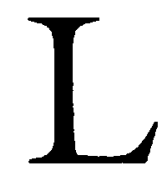

A méthode classique de protection du myocarde des conséquences de l'ischémie durant la chirurgie à coeur ouvert est l'arrêt avec cardioplégie froide. Or plusieurs ne considèrent plus cette méthode comme offrant les meilleurs résultats. L'arrêt avec cardioplégie chaude ${ }^{1}$ ainsi que la chirurgie coronarienne sans recours à la $\mathrm{CEC}^{2,3}$ sont des procédures communes dans plusieurs centres. L'utilisation des activateurs du plasminogène tissulaire et/ou l'angioplastie en urgence ont révolutionné le traitement non chirurgical de l'occlusion coronarienne aiguë. ${ }^{4}$ Ces développements cliniques sont survenus en même temps que s'améliorait notre compréhension des mécanismes sous jacents aux lésions cellulaires liées à la reperfusion myocardique. ${ }^{5,6}$ On examine actuellement plusieurs stratégies innovatrices, pharmacologiques et non pharmacologiques, destinées à accroître la protection myocardique avant l'ischémie et à limiter les lésions lors de la reperfusion suite à une ischémie. Cette mise à jour passe en revue les mécanismes et l'expérience clinique acquise à ce jour concernant quatre de ces interventions, qui intéressent particulièrement l'auteur.

\section{Modification du métabolisme énergétique}

Le coeur est un tissu omnivore capable d'utiliser comme substrats énergétiques les acides gras libres, le glucose, le lactate et le pyruvate. ${ }^{7}$ Lors de conditions aérobiques normales, les acides gras libres représentent la principale source d'énergie pour le myocarde. Si on prend à titre d'exemple l'oxydation du palmitate, un acide gras à 16 carbones, on obtient 129 molécules d'adénosine triphosphate (ATP), ce qui représente un rendement d'ATP par atome d'oxygène utilisé de 2,83. Le glucose, l'autre combustible majeur utilisé par le coeur, est d'abord métabolisé en pyruvate, par un procédé appelé glycolyse, pour ensuite entrer dans le cycle de l'acide citrique sous forme d'acétyl CoA et subir une oxydation complète jusqu'au $\mathrm{CO}_{2}$ et $\mathrm{H}_{2} \mathrm{O}$. La décarboxylation du pyruvate pour former de l'acétyl CoA est catalysée par le complexe pyruvate déshydrogénase $(\mathrm{CPD})$. Le métabolisme aérobique du glucose produit 36 molécules d'ATP, représentant un rendement d'ATP par atome d'oxygène utilisé de 3,17. En l'absence d' $\mathrm{O}_{2}$, le pyruvate est transformé en lactate, par la glycolyse anaérobique, de sorte que, dans ces conditions, le rendement d'une molécule de glucose qui entre dans la voie glycolytique n'est que de deux molécules d'ATP.

Durant l'ischémie sans débit coronarien, le coeur utilise uniquement le métabolisme anaérobique du glucose pour sa production d'ATP. Durant cette période d'absence de flot, la seule source de glucose est la molécule d'entreposage du glucose, le glycogène, et une molécule de glycogène métabolisée en lactate produit trois molécules d'ATP. L'énorme différence entre la production d'énergie dans un environnement aérobique plutôt qu'anaérobique souligne l'urgence de rétablir le flot sanguin et par.le fait même le métabolisme oxydatif aux cellules myocardiques. En outre, l'épargne en $\mathrm{O}_{2}$ du glucose par rapport aux acides gras libres suggère que l'utilisation préférentielle du glucose au cours de la reperfusion qui suit une ischémie devrait être bénéfique. On a montré dans plusieurs études in vitro ${ }^{8,9}$ qu'on améliorait la fonction ventriculaire en favorisant l'oxydation du glucose lors de la reperfusion suite à une ischémie.

Même si la glycolyse est essentielle durant une ischémie sans flot sanguin, c'est une arme à deux tranchants qui peut causer des dommages si utilisée de façon incontrôlée durant l'ischémie et la reperfusion. Lorsque des molécules d'ATP produites par glycolyse sont utilisée par les cellules, des ions $\mathrm{H}+$ sont produits. $\mathrm{La}$ cellule échange ensuite ces ions $\mathrm{H}+$ pour des ions $\mathrm{Na}+$ via l'échangeur $\mathrm{Na}+/ \mathrm{H}+$ dans un effort de maintien du $\mathrm{pH}$ cellulaire. Par conséquent, durant l'ischémie, mais surtout durant la reperfusion, il se produit un gain net d'ions $\mathrm{Na}+$ dans la cellule. ${ }^{10}$ Une augmentation du $\mathrm{Na}+$ intracellulaire active le mécanisme d'échange $\mathrm{Na}+/ \mathrm{Ca}++.{ }^{11}$ Cette entrée excessive de $\mathrm{Ca}++$ a été mise en cause comme étant un des processus clefs entraînant la lésion myocardique. Une augmentation du $\mathrm{Ca}++$ intracellulaire entraîne une entrée et une sortie accrues du Ca++ dans le réticulum sarcoplasmique et un emmagasinement plus grand du $\mathrm{Ca}++$ dans les mitochondries, deux processus qui gaspillent l'ATP, stimulent l'activation de la phospholipase et par conséquent, entraînent des dommages aux membranes. ${ }^{5}$

Lorsqu'existent des conditions de stress, comme durant la chirurgie cardiaque ou suite à un infarctus, les niveaux d'acides gras libres sont augmentés et l'oxydation du glucose est inhibée. ${ }^{12}$ Le découplage de l'oxyda- 
tion du glucose de la glycolyse peut être dommageable puisqu'il augmente la production d'ions $\mathrm{H}+$ et réduit la production efficiente d'ATP. Le fait d'altérer le métabolisme myocardique durant l'ischémie en prévenant la glycolyse excessive et, durant la reperfusion, en favorisant l'oxydation du glucose et en améliorant le couplage entre cette oxydation du glucose et la glycolyse, améliore la récupération fonctionnelle suite à une ischémie chez des modèles animaux d'ischémie globale. ${ }^{13}$ Des études cliniques, chez des patients souffrant de maladie cardiaque ischémique, de la ranolazine, un médicament qui stimule l'oxydation du glucose en augmentant l'activité du CPD, ont montré que même si le médicament améliore la fonction diastolique chez des patients ayant déjà fait un infarctus, il ne semble pas efficace pour réduire la survenue ou la sévérité de l'angine. ${ }^{15}$ Le rôle, chez l'homme, des stimulants du CPD dans la réduction du dommage cellulaire associé à la reperfusion fait toujours l'objet de recherches.

\section{Préconditionnement ischémique}

Murry et al. ${ }^{16}$ ont décrit en 1986 pour la première fois le phénomène du préconditionnement ischémique (PCI), un procédé par lequel des épisodes ischémiques brefs et répétitifs induisent une protection temporaire contre le dommage myocardique résultant d'une période d'ischémie subséquente plus prolongée. Ce mécanisme protecteur important est associé à une réduction de la taille de l'infarctus suite à une ischémie régionale, ${ }^{17}$ à une reprise de la fonction mécanique améliorée durant la reperfusion suivant une ischémie globale secondaire à une absence de flot ${ }^{18}$ ainsi qu'à une diminution des dysrythmies induites par l'ischémie. ${ }^{19}$ Le PCI améliore le couplage entre l'oxydation du glucose et la glycolyse et protège le coeur des conséquences d'une ischémie globale sans flot. ${ }^{18}$ Même si l'ischémie est le stimulus de préconditionnement classique, il est maintenant apparent que plusieurs autres interventions peuvent servir de préconditionnement. Beaucoup de preuves se sont accumulées impliquant l'adénosine comme facteur déclanchant ou comme médiateur du PCI. ${ }^{20,21}$ La stimulation du récepteur $\mathrm{A}_{1}$ de l'adénosine mime le $\mathrm{PCI}^{22}$; l'effet protecteur du PCI est diminué par les antagonistes du récepteur $\mathrm{Ado}^{17}$ et facilité ou par l'augmentation de la relâche d'Ado, ${ }^{23,24}$ ou par l'inhibition de sa destruction. ${ }^{25}$ D'autres mécanismes du PCI ont été proposés; il s'agit d'une augmentation de la synthèse de la protéine active dans le choc thermique, ${ }^{26}$ d'une augmentation de l'activité des canaux $\mathrm{K}+$ dépendants de l'ATP, ${ }^{27}$ d'une réduction de l'utilisation d'ATP, ${ }^{16}$ et d'une augmentation de l'activité de la kinase protéique $C^{28}$ Une protection de type PCI a aussi été notée suite à l'administration de bradykinine et de catécholamine. ${ }^{28,29}$
Les protocoles de PCI utilisés chez les patients subissant une angioplastie sont associés à une réduction de l'incidence des ectopies ventriculaires durant l'occlusion coronarienne ${ }^{30}$ et à une réduction des modifications du segment ST durant l'inflation du ballon. ${ }^{31}$ De façon analogue, chez des patients subissant une chirurgie à coeur ouvert, un protocole de PCI comprenant deux périodes de trois minutes chacune de clampage aortique espacées d'une période de reperfusion de 2 minutes et suivies d'une période d'ischémie de 10 minutes, a permis de démontrer un taux réduit de déplétion d'ATP et de relâche de troponine $\mathrm{T}$ comparable aux coeurs contrôles. ${ }^{32}$ Dans les procédures chirurgicales de revascularisation myocardique qui se font sans utiliser la CEC, le PCI est souvent réalisé au niveau du vaisseau cible pour conférer une protection durant la période sans flot que représente l'anastomose chirurgicale.

\section{Adénosine}

Dans des modèles animaux d'ischémie régionale ${ }^{34}$ et globale, ${ }^{13}$ l'adénosine endogène, de même que l'adénósine exogène, protègent le coeur des conséquences de l'ischémie, entraînant une réduction marquée de la taille de l'infarctus ${ }^{33}$ et une amélioration de la récupération de la fonction mécanique durant la reperfusion.

L'adénosine peut agir par plusieurs voies protectrices, qui sont la dilatation des coronaires et des collatérales, responsables d'une augmentation de l'apport de $\mathrm{O}_{2}$, la modulation de l'activité des catécholamines, l'activation de la protéine $\mathrm{C}$ kinase, l'ouverture des canaux $\mathrm{K}+\mathrm{ATP}$ (qui entrainent des effets inotropes et chronotropes négatifs et diminuent la demande en $\mathrm{O}_{2}$ ), et l'inhibition de la cytotoxicité des neutrophiles et de l'adhésion plaquettaire dans la microcirculation coronarienne. ${ }^{35}$ En conditions aérobiques, l'adénosine inhibe la glycolyse, favorise le couplage entre la glycolyse et l'oxydation du glucose et réduit la production d'ions $\mathrm{H}+$ provenant du métabolisme du glucose. ${ }^{13}$

Il y a, à date, une étude clinique publiée qui évalue les effets de l'administration d'adénosine chez des patients subissant une chirurgie cardiaque. Mentzer et al. ${ }^{36}$ ont noté, par rapport à des patients témoins, une réduction des besoins en inotropes et une amélioration de la fraction d'éjection des patients qui ont reçu 140 $\mu \mathrm{mol} \cdot \mathrm{kg}^{-1} \cdot \mathrm{min}^{-1}$ d'adénosine immédiatement avant la CEC et qui ont reçu des solutions de cardioplégie froide avec sang auxquelles ont avait ajouté de $500 \mu \mathrm{m}$ à $2 \mathrm{mM}$ d'adénosine. L'administration d'adénosine était aussi associée à une réduction significative des besoins de sang et de ses dérivés. ${ }^{37}$ Chez les patients subissant une angioplastie, une infusion intracoronarienne d'adénosine à la dose de $2 \mathrm{mg} \cdot \mathrm{min}^{-1}$ durant 10 minutes avant 
l'angioplastie percutanée transluminale réduisait de façon significative la modification des segments ST et les douleurs thoraciques associées au gonflement initial du ballon lorsque comparé à des sujets témoins traités par placebo. ${ }^{38}$ Ces données préliminaires sont excitantes et suggèrent que le prétraitement à l'adénosine peut être approprié dans les procédures cardiaques électives où le coeur est soumis à des situations d'ischémie-reperfusion.

\section{Agents anesthésiques}

Les anesthésiques volatils, en particulier l'halothane et l'isoflurane, semblent posséder des propriétés cardioprotectrices. Dans les modèles animaux d'ischémie régionale et globale, les deux anesthésiques ensemble ou isolément, à condition d'avoir été introduits avant ou durant l'épisode ischémique, améliorent la fonction ventriculaire gauche post ischémie, ${ }^{39}$ limitent la sidération myocardique, ${ }^{40}$ diminuent les dysrythmies de reperfusion $^{41}$ et diminuent la taille des infarctus. ${ }^{42}$ L'efficacité de l'halothane et de l'isoflurane à limiter la dysfonction ischémique apparaît moins certaine si ces agents sont introduits uniquement au moment de la reperfusion. ${ }^{43}$

L'halothane diminue la contractilité et la consommation d'oxygène de façon plus marquée que l'enflurane ou l'isoflurane, sans altérer le flot coronarien et la livraison d'oxygène. ${ }^{44} \hat{A}$ une concentration inférieure à une CAM, l'isoflurane augmente le flot coronarien et de ce fait la livraison d'oxygène, tout en ayant des effets limités sur la contractilité. ${ }^{45}$ Les agents volatils produisent des effets similaires sur la balance entre l'offre et la demande en $\mathrm{O}_{2}$ chez les humains atteints de maladie coronarienne. ${ }^{46}$ Les agents volatils économisent les niveaux de phosphate à haute énergie dans le myocarde. L'halothane améliore la restauration du contenu en ATP du myocarde lors de la reperfusion, ${ }^{48}$ diminue la relâche de lactate durant l'ischémie et diminue la dégradation de l'ATP en adénosine et inosine. ${ }^{49} \mathrm{~L}$ 'isoflurane préserve aussi le contenu en ATP du myocarde dans des coeurs soumis à une ischémie et à une reperfusion. Il existe une solide évidence à l'effet que les agents volatils peuvent aider le myocarde à normaliser le cycle pathologique du $\mathrm{Ca}++$ durant la reperfusion. L'halothane en particulier diminue l'accumulation $\mathrm{du} \mathrm{Ca}++\mathrm{du}$ myocyte ${ }^{50} \mathrm{en}$ réduisant directement la relâche du $\mathrm{Ca}++$ à partir du réticulum sarcoplasmique ${ }^{51}$ et en inhibant le mécanisme d'échange $\mathrm{Na}+\mathrm{Ca}++$ pour prévenir l'influx de $\mathrm{Ca}++.{ }^{52}$

\section{Conclusion}

L'amélioration de notre compréhension des réponses cellulaires à l'ischémie et à la reperfusion suite à une ischémie à permis le développement de plusieurs stratégies nouvelles et excitantes pour limiter les conséquences néfastes de l'ischémie myocardique prolongée. L'utilisation clinique de ces connaissances apporte l'espoir de pronostics améliorés pour les patients souffrant de maladie coronarienne.

\section{Références}

(Voir page R34) 